\title{
Tangence
}

\section{De la peinture à l'écriture dramatique : vers une diffraction du sens}

\section{Marie-Christine Lesage}

Numéro 46, décembre 1994

Un théâtre de passage

URI : https://id.erudit.org/iderudit/025842ar

DOI : https://doi.org/10.7202/025842ar

Aller au sommaire du numéro

Éditeur(s)

Tangence

ISSN

0226-9554 (imprimé)

1710-0305 (numérique)

Découvrir la revue

Citer cet article

Lesage, M.-C. (1994). De la peinture à l'écriture dramatique : vers une diffraction du sens. Tangence, (46), 82-96. https://doi.org/10.7202/025842ar d'utilisation que vous pouvez consulter en ligne.

https://apropos.erudit.org/fr/usagers/politique-dutilisation/ 


\section{De la peinture à l'écriture dramatique: vers une diffractation du sens \\ Marie-Christine Lesage}

Depuis 1980, le théâtre québécois n'a cessé de glisser vers de nouvelles formes et de nouveaux contenus, transgressant un système acquis et déterminé, pour s'intéresser à d'autres hypothèses, et bousculer ainsi toute une tradition. Si tout nouveau style artistique met au jour des transformations de la société dans l'ordre de la connaissance, les formes théâtrales présentes rendent compte, semble-t-il, d'une modification profonde dans les processus d'appréhension et de représentation du réel, à partir desquels les artistes fondent un nouveau style. Le théâtre actuel, côté texte et côté scène, pose aux spectateurs et aux divers interprètes un problème ardu de lisibilité. La question qu'il met en vue concerne ainsi moins une perte du contenu que la capacité et l'aptitude réelle à interpréter, lire et décoder adéquatement les ouvres inédites qui sont présentées. La question du contenu s'associe étroitement au problème du renouveau de la communication entre le spectateur et le théâtre tel qu'il s'écrit et se pratique aujourd'hui.

On associe fréquemment les nouvelles formes théâtrales au "théâtre de l'image", théâtre pictural et plastique qui accorde une place prépondérante à la matière, telle que la lumière ou les objets, dans la construction du sens. Aussi, c'est sur la scène et dans l'ordre du visuel qu'ont eu lieu les premiers changements du théâtre québécois, alors qu'il échappe radicalement à toute la civilisation de la pensée et du livre dans laquelle il a longtemps été enchâssé: à ce titre, Carbone 14, Robert Lepage, le Théâtre Repère, le Théâtre Ubu ainsi qu'Omnibus constituent les emblèmes de ce théâtre de l'image. Ce dernier, délaissant les structures linéaires et réalistes de représentation, fabrique des univers où le sens se trame de façon analogique et transversale, ce qui risque fort de déstabiliser le spectateur accoutumé à une ancienne façon de les interpréter. Ces troupes ont élaboré des spectacles sans avoir recours au texte dramatique traditionnel ${ }^{1}$, s'inspirant plutôt

1 Lire à ce sujet l'article de Louise Vigeant, "Le théâtre avec ou sans drame", Jeu, $\mathrm{n}^{\circ} 53$, décembre 1989 , p. 27-31. 
de la peinture ${ }^{2}$, du cinéma, du travail avec la lumière pour élaborer ce qu'on a nommé une "écriture scénique" ${ }^{3}$. C'est dans cette seule zone d'expérimentation que le théâtre fait un bond qualitatif, qu'il redéfinit le rapport texte/scène, en cherchant à développer un nouveau langage où le logos et le mode de communication propre au texte hiérarchisé et linéaire ne forment plus nécessairement l'inférence créatrice principale.

Un nouveau style théâtral est donc né sur la scène québécoise des années quatre-vingt, manifestant non seulement une nouvelle vision, mais un nouveau langage, une nouvelle façon de faire sens. Mais le théâtre étant le fruit d'une relation entre le texte et la scène - relation qui a pu se constituer d'éloignements et de confrontations ces dernières années -, il y a lieu de se demander si ces deux axes qui fondent le théâtre occidental ont vécu, depuis une décennie, un bouleversement similaire. Si le pôle de la scène a activé l'évolution du théâtre vers de nouveaux horizons de sens, s'est-il également créé un texte de l'image? Autrement dit, est-il possible de déceler une conjonction dans le mode actuel de création du texte et de la scène? Ce serait le signe que le renouvellement du théâtre québécois dans son ensemble traduit et participe à une transformation de la société dans l'ordre de la connaissance. Si la nouvelle logique artistique analogique survint d'abord sur la scène, c'est que cette dernière contenait les éléments naturels au déploiement d'une communication visuelle ayant ses propres articulations, dégagées du texte dramatique traditionnel. D'autre part, la prééminence accordée à la forme visuelle par le théâtre contemporain prend essentiellement racine dans le changement de point de vue que celui-ci opère face à la réalité, et non seulement dans une tendance formaliste. En conséquence, j'aimerais montrer ici que les nouvelles écritures dramaturgiques participent également à ce déplacement de point de vue sur le monde, en empruntant un langage analogique semblable à celui de l'écriture scénique actuelle.

2 La notion de scénographie tire son origine entre autre de la peinture: "À partir de la Renaissance, le mot - emprunté à Vitruve - désigne l'art de la perspective, l'art du point de vue, appliqué à la peinture, à l'architecture, à la ville". "Scénographie", Dictionnaire encyclopédique du thêâtre, Paris, Bordas, p. 755 .

3 En ce qui a trait à cette nouvelle écriture scénique, voir l'article de Chantal Hébert et Irène Perelli-Contos, "Une mutation en cours "dans Théatre/ Public, no 117 , p. 64-73. Également, C. Hébert, "L'écriture scénique actuelle: l'exemple de Vinci" dans Nuit blancbe, $\mathrm{n}^{\circ}$ 55, Printemps 1994, p. 54-58. 
84

\section{Sur la trace d'une nouvelle écriture}

À cet effet, je prendrai pour modèle Le syndrome de Cézanne ${ }^{4}$ de Normand Canac-Marquis comme point de repère du langage dramaturgique en gestation. Ainsi que l'a écrit Paul Lefebvre, cette pièce signale effectivement un changement d'état dans le système dramaturgique québécois :

Quant au Syndrome de Cézanne (1987) de Normand CanacMarquis, il marque une nouvelle tendance dramaturgique. Depuis les années où l'œuvre de Dubé est passée des cuisines populaires aux salons bourgeois, les pièces québécoises étaient habituellement fondées sur un conflit unique et évident [...] et le travail du spectateur consistait en la découverte des conséquences de cette action sur les personnages. Le syndrome de Cézanne renverse cette construction. ${ }^{5}$

D'abord, cette cuvre propose une vision qui innove en regard de la dramaturgie traditionnelle, par la présentation d'une structure dégagée des contraintes de la mimésis. Rappelons simplement que le système de représentation traditionnel allie un point de vue centré sur la réalité extérieure des événements (qu'ils soient sociopolitiques ou psychologiques), à un ordre logique valorisant les opérations déductives de la pensée: toutes les actions doivent s'expliquer rationnellement, ne laissant que très peu de place au hasard et à l'interprétation subjective. Cet alliage concourt à édifier l'impression de vraisemblance chez le spectateur, alors qu'en fait il correspond à un système illusionniste ancré dans des conventions acquises, et satisfaisant à certaines habitudes intellectuelles propres à un moment daté de la culture. Érigé en un tout stable et normatif, ce système échange aujourd'hui moins d'informations nouvelles avec son environnement. D'autant plus que les auteurs et les metteurs en scène québécois ne cessent de mettre de l'avant - depuis plus d'une décennie - l'idée que le rôle de l'art, et plus particulièrement du théâtre, ne se réduit pas à une reproduction extérieure du réel. La création théâtrale remet en question le mode linéaire de transmission d'une vision ${ }^{6}$.

4 Normand Canac-Marquis, Le syndrome de Cézanne, Montréal, Les Herbes rouges, 1988 .

5 "Le réel des rêveurs: regard sur les années quatre-vingt", Veilleurs de nuit, $\mathrm{n}^{\circ} 2,1989-1990$, p. 124.

6 Et notre difficulté à interpréter et apprécier parfois ce théâtre vient du fait que nous demeurons pour une bonne part encore prisonniers du thêâtre réaliste et mimétique. 
Mais ce questionnement a été amorcé - et se poursuit dans de nombreuses œuvres dramaturgiques plus récentes - bien avant Le syndrome de Cézanne. Comme on le sait, tout passage d'un système artistique à un autre suit un cheminement de destruction et de réinterprétation d'éléments appartenant à l'ancien modèle de création. Au début des années quatre-vingt apparaissent de nouveaux auteurs dramatiques qui vont, dans un premiers temps, jouer le rôle de pionniers-destructeurs. C'est le cas, entre autres, de René-Daniel Dubois et Normand Chaurette, dont les pièces 26 bis, impasse du Colonel Foisy et Provincetoun Playbouse, juillet 1919, javais 19 ans apparaissent comme les jalons de cette première période alliant destruction des sujets et réinterprétation de la structure traditionnelle du théâtre québécois. Ces deux pièces, par des procédés de mise en abyme, par l'incursion de la voix de l'auteur et de la figure de l'artiste, vont mettre à bas toute la logique propre à la dramaturgie réaliste (ou illusionniste); elles empêchent le spectateur de s'identifier à ce qui se déroule devant lui, en délinéarisant et en complexifiant la structure dramatique, en questionnant ouvertement le théâtre dans sa façon de communiquer le réel et l'imaginaire. Elles accentuent la fonction métacommunicative ${ }^{7}$, en empêchant ainsi la mise en place d'une histoire continue et en faisant plonger le spectateur dans le monde individuel et intérieur de l'artiste face à sa création ${ }^{8}$. Ces deux revirements - perte du sujet et de l'histoire, ainsi que passage du monde extérieur au monde intérieur - vont se retrouver dans toute la dramaturgie subséquente. Si l'écriture thêâtrale de ces deux auteurs s'articule d'abord autour de la destruction d'un ancien ordre, elle présente aussi les germes d'une nouvelle tendance à venir. Pionniers, ils le sont en ouvrant la porte à de nouvelles possibilités de sens et d'écriture, vite assimilées par leurs successeurs. Si certaines pièces écrites depuis perpétuent la destruction de l'ancien système, tout en demeurant centrées sur une autoréférentialité le plus souvent autocritique $^{9}$, d'autres, encore rares, semblent amorcer concrètement le

7 Voir à ce sujet l'article de Gilles Deschâtelets, "L'année de tous les miroirs ", Veilleurs de nuit, $\mathrm{n}^{\circ} 2,1989-90$, p. 56-62; cet article approfondit la question du thêâtre autothématisé.

8 Sur la question de la figure du créateur, je renvoie le lecteur aux articles de Louise Vigeant, "Du réalisme à l'expressionnisme: la dramaturgie récente à grands traits", Jeu, $\mathrm{n}^{\circ}$ 58, mars 1991, p. 7-16; également, Paul Lefebvre, "Le réel des rêveurs: regard sur les années quatre-vingt ", op. cit., p. 117-129.

9 Et je pense ici à La répétition de Dominic Champagne, Billy Strauss de Lise Vaillancourt, J'écrirai bientôt une pièce sur les nègres de Jean-François Caron, 
86

passage vers un nouvel ordre artistique. C'est à cet égard que Le syndrome de Cézanne retient notre attention ${ }^{10}$. Cette œuvre constitue, à notre avis, le premier jalon susceptible de diriger vers une meilleure compréhension de cette tendance dramaturgique; elle en est une passionnante clé d'entrée.

La pièce met en scène un homme de la mi-trentaine nommé Gilbert, qui tente de "réparer un char écrasé par un truck fantôme "11. Cette réparation va en fait consister en une lente remontée mnésique du protagoniste vers un événement survenu un 16 juillet 1986, dont on n'apprendra la teneur qu'à la fin: un accident d'automobile dans lequel a péri Suzanne, sa compagne, ainsi que leur enfant. Les titres des scènes comportent tous un lien quelconque avec l'automobile ${ }^{12}$, et correspondent, symboliquement, aux états intérieurs du personnage; elles ne s'ordonnent ni temporellement ni déductivement les unes par rapport aux autres, mais suivant une cohérence propre à l'esprit de Gilbert. Autrement dit, le lecteur a accès à un réel traité selon la logique intérieure du protagoniste, qui retrace progressivement l'image d'un événement antérieur, par le biais de retours sur le passé, de dialogues imaginaires avec Suzanne, de rêves, ou encore de conversations téléphoniques avec un inspecteur. Cet échantillonnage de fragments de réalité auquel procède sa pensée va déterminer, à l'intérieur d'une scène et entre les scènes,

pour ne nommer que ceux-là. Ces textes posent de façon intelligente la problématique du théâtre québécois actuel, que ce soit du côté du jeu de l'acteur, de la création de l'auteur, de son rapport à l'univers social et politique, etc.

10 Le choix des oeuvres n'est pas motivé uniquement par la période temporelle, mais plutôt par l'observation d'une logique artistique similaire déployée à I'intérieur de certains textes. Aussi, un même auteur pourra avoir écrit, après 1980, des oeuvres qui n'appartiennent pas toutes à la nouvelle tendance dramaturgique. Ainsi en est-il, par exemple, de la différence entre Le syndrome de Cézanne et Les jumeaux d'Urantia de Normand Canac-Marquis ; cette dernière, quoique mettant en scène la figure du créateur, préserve une construction relativement traditionnelle. On retrouve des cas semblables dans les oeuvres de René-Daniel Dubois et de Michel-Marc Bouchard. La plupart des auteurs récents ont écrit des textes qui appartiennent aux deux systèmes artistiques, l'ancien et celui en train de se réaliser; cela appuie l'idée voulant que la dramaturgie actuelle se situe dans une période de passage entre deux visions. Il y a donc mélange entre l'ancien et le nouveau.

11 Normand Canac-Marquis, op. cit., p. 18.

12 Par exemple: "Prestone", "Cédez/Yield", "Allumage ", "Pare-soleil ", "R.P.M. (Rotations par minutes)", etc. 
une vue non pas logique mais analogique de l'événement; des images et des éléments issus de contextes non homogènes (appartenant à des temps et à des situations différentes) vont s'agglomérer sur la base de leur seule relation à un même objet, soit l'accident. Par exemple, la huitième scène se structure à l'image de l'emballement intérieur de Gilbert. Celui-ci entremêle les voix de l'inspecteur Wancikovski, d'un C.B. de police, de Suzanne et de lui-même. Ce mixage va donner lieu à un dialogue qui associe - à l'image du flot de la conscience du protagoniste - des fragments disparates de réel ayant tous un lien quelconque - à découvrir par le lecteur - avec l'accident:

THOMAS WANCIKOVSKI

Donnez position. Dix quat'.

VOITURE 30-07

Toujours la même position, sortie 17 , kilométrage 17 , qui qui parle? Dix quat'.

THOMAS WANCIKOVSKI

Capitaine Wancikovski. Thomas... Thomas Wancikovski...

Vous comprenez? Dix quat'.

\section{GILBERT}

Wancikovski. Oui, j'ai compris.

ELIE

Gilbert... L'auto! Qu'est-ce qui est arrivé à l'auto?

\section{GILBERT}

Les clés sont dans la sacoche de la madame qui meurt dans le trafic. Fouille dedans!

Cet agencement s'accorde à la logique du rêve, notamment par la réunion et la concentration dans un même espace mental de ce qui est normalement dispersé dans l'espace et dans le temps. Ainsi en est-il du lien qu'entretiennent les scènes entre elles: toute la pièce se compose d'une mosaïque d'images qui se répondent en écho autour de l'événement ; cette mosaique s'articule en une succession de boucles et d'aller-retour qui traduisent la relation mouvante qu'entretient la perception subjective de Gilbert avec le monde extérieur. Nous plongeons ainsi dans une conception du mouvement intérieur dépouillé de toute ordonnance temporelle. De cette façon, la pièce décentre son point de vue de l'événement dramatique, pour insister sur l'effet de conscience et sur le processus de construction d'une image intérieure du monde. Nous y reviendrons plus loin. 
88

À la lecture, Le syndrome de Cézanne révèle trois niveaux possibles de compréhension, lesquels vont du plus simple au plus complexe. À un premier degré d'interprétation, le lecteur ne retiendra que la relation de couple - sujet traditionnel de la dramaturgie psychologique - entre Gilbert et Suzanne, relation en crise qui se dirige vers l'éclatement. L'accident devient ainsi la métaphore de l'effondrement de ce couple. À un second niveau, le schéma de la pièce suit celui de l'enquête policière. Il y a en effet un inspecteur, Thomas Wancikovski, chargé d'enquêter sur les mobiles de l'accident, mais il n'apparaît que de façon fugitive, exception faite des dernières scènes. Le schéma de l'enquête se déroule en deux temps. Les huit premières scènes installent un système d'informations indicielles, relatives à l'événement, qui surgissent, comme nous l'avons dit plus haut, de sources diversesd'un rêve, d'un souvenir, etc. - ; le lecteur a donc la charge de se construire une vue cohérente de l'événement à partir de ces indices épars. Les scènes subséquentes mettent en place un système d'hypothèses plausibles quant aux causes de l'événement, que le lecteur doit déduire des scènes présentées avant de choisir. Par exemple, les scènes 10 et 15 exposent des versions distinctes du matin du 16 juillet: dans la première version Gilbert et Suzanne se disputent à propos d'argent et elle lui annonce qu'elle rompt, alors que dans la seconde, ils sont amoureux. Selon le point de vue que privilégiera le spectateur, la cause plausible de l'accident qu'il retiendra sera différente (meurtre, suicide ou hasard). Aussi, c'est à juste titre que Carole Fréchette écrivait:

[...] peut-être à cause de sa structure complexe, Le syndrome de Cézanne ne provoque pas l'adhésion immédiate du spectateur. Lorsque prend fin cette histoire de mort et d'amour brisé, on demeure pensif. On continue pendant un moment à jouer avec les morceaux du casse-tête et l'on ne découvre que peu à peu l'ampleur de l'iceberg caché sous la pointe. Le syndrome de Cézanne est un texte à retardement, qui explose après coup ${ }^{13}$.

La complexité de la pièce s'éclaire quelque peu lorsqu'on examine le troisième niveau d'interprétation, qui englobe et explique la structure des deux autres. Ce troisième aspect est essentiellement métacommunicatif ${ }^{14}$, c'est-à-dire que l'auteur communique au lec-

13 Carole Fréchette, "Le syndrome de Cézanne: une voix de métal ", Jeu, $\mathrm{n}^{\circ}$ 46, 1988, p. 85.

14 Le concept de métacommunication se définit comme un discours sur la communication, c'est-à-dire "lorsque nous ne nous servons plus de la communi- 
teur - qui fait l'effort de prendre la clé tendue - une hypothèse pour saisir le mode de communication de l'œuvre. Celle-ci nous est indiquée essentiellement par le titre et la deuxième scène. $\mathrm{Si}$ dans les pièces qui détruisent l'ordre de l'ancien système artistique, la fonction de métacommunication est intrinsèque aux œuvres (elle en justifie et en tisse une bonne partie du sens), dans Le syndrome de Cézanne cette fonction devient externe (elle se déplace vers la relation entre l'œuvre et le spectateur); elle agit alors comme guide et indice d'une nouvelle vision et d'un nouveau langage ${ }^{15}$. Par son titre, qui fait référence à la peinture de Cézanne, cette ouvre propose effectivement une entrée vers une meilleure compréhension de tout un pan de la dramaturgie québécoise actuelle.

Le syndrome de Cézanne nous informe sur l'importance accordée aux divers états pathologiques du protagoniste, plutôt que sur leur cause. La pièce déplace ainsi son point de vue de l'objet de la sensation - c'est-à-dire de l'événement extérieur qui forme le sujet traditionnel de la dramaturgie - vers la sensation elle-même saisie à l'état brut dans l'espace intime de la perception du personnage. Et c'est ici que la désignation cézannienne du syndrome prend toute sa valeur. La deuxième scène intitulée "Destination" peut se lire à la fois comme l'exposition du sujet réel de la pièce et comme le lieu où l'on doit se rendre ${ }^{16}$. Elle se

cation pour communiquer, mais pour communiquer sur la communication", P. Watzlawick et al., Une logique de la communication, Paris, Seuil, 1972, p. 35. Ainsi, la dramaturgie métacommunique lorsque les auteurs ne se servent plus du texte uniquement pour communiquer de l'imaginaire ou une situation fictive, mais font de la fiction thêâtrale, du texte et du théâtre luimême l'objet de leur questionnement, interrogeant certains de ses aspects. Cette métacommunication peut être implicite, incluse dans la structure même de la pièce (c'est le cas des mises en abymes), ou explicite, c'est-à-dire faisant l'objet d'un questionnement discursif.

15 Il convient ici de préciser que la pièce de Normand Canac-Marquis constitue un indicateur qui permet de fouiller certaines pistes qui n'ont pas nécessairement été pensées par l'auteur; sa richesse vient des possibilités interprétatives qu'elle suscite. D'autres pièces écrites par la suite procèdent également de cette nouvelle logique artistique, telles $L a$ déposition d'Hélène Pedneault et Celle-là de Daniel Danis; toutes deux présentent en effet de nombreux points en commun avec Le symdrome de Cézanne en ce qui a trait à leur relation au contenu et au mode de composition. Je me propose de faire cette étude comparative dans le cadre de ma thèse de doctorat.

16 La phrase qui suit le titre indique: "En conduite préventive, une des règles est de bien connaitre sa destination et le parcours pour s'y rendre " (26), ce qui est assez explicite quant à l'intention de cette scène. 
90

résume à un paragraphe où l'inspecteur parle de la façon de peindre de Cézanne:

Cézanne représente la réalité en supprimant volontairement les règles classiques de la profondeur et des dimensions. Il oppose au réalisme une réalité différente qui donne au sujet sa raison profonde d'être. Tous les objets qui entourent le modèle, qu'il s'agisse d'une chaise, d'une porte, d'une tasse à café et même la cuillère de cette tasse à café... tous ces détails livrent une perception fugitive et différente de la réalité. Ce qui importe chez lui, ce n'est pas tant le sujet observé que la conscience de celui qui observe.

Les références et les allusions à la peinture de Cézanne ${ }^{17}$ constituent autant de messages métacommunicatifs dont la substance se loge au sein de la phrase: "Ce qui importe chez lui, ce n'est pas tant le sujet observé que la conscience de celui qui observe". Le syndrome de Cézanne amorce donc une recherche similaire à celle entreprise par Cézanne dans sa tentative de "saisir aussi directement que possible les données pures des sens et de la conscience "18. Le dévoilement de cette clé par l'inspecteur indique à l'évidence que toute plongée dans l'espace sensoriel et mnésique adopte nécessairement la forme d'une investigation: l'enquête policière représenterait simplement le mode de fonctionnement de la conscience, toute perception intérieure consistant en une recherche suivie sur le monde. Le schéma de l'enquête - qui se retrouve également dans La déposition d'Hélène Pedneault répondrait ainsi à la logique du réel interne, qui fonctionne par échantillonnage de fragments de réel surgissant d'abord dans le désordre, puis s'unissant selon un ordre d'affinités et de corrrespondances analogiques plutôt que logiques.

Bref, ce troisième niveau a pour objet d'indiquer le point de vue perceptif adopté par la pièce, qui ne peut se com-

17 À la scène 12, une didascalie indique que Gilbert "trouve un livre sur Cézanne qu'il lira durant la scène $13 *(70)$; à la scène 14, Gilbert, en parlant de Suzanne à l'inspecteur, dit qu'elle avait "un amour inconditionnel pour Cézanne. (81); à la scène 15 , Gilbert fait une allusion qui renvoie à la peinture: "j'ai toujours eu le nez à deux pouces et quart de la toile, pis j'ai jamais compris ce que ça voulait dire ces taches-là [...]" (88); et enfin, à la scène 16 , la pièce se termine sur l'inspecteur qui prend le livre sur Cézanne et lit: "mes yeux sont tellement collés au point de vue que je regarde qu'il me semble qu'ils vont saigner. (93).

18 Pierre Francastel, Peinture et société: naissance et destruction d'un espace plastique: de la Renaissance au cubisme, Lyon, Audin Êditeur, 1951, p. 175. 
prendre qu'en empruntant le chemin de l'hypothèse analogique ou de l'abduction ${ }^{19}$ engendrée par le mixage entre la peinture de Cézanne et le mode de composition du Syndrome de Cézanne. Cette convocation d'un mode de création pictural comme modèle structurant de l'écriture dramatique n'est pas le fruit du hasard: la peinture moderne procurerait-elle un nouveau point de vue sur le monde que le théâtre assimilerait actuellement? Il est possible que les arts visuels aient déjà amorcé, au début du siècle, cette transformation dans la pensée artistique, et ce bien avant le théâtre. Aussi, pour nous aider à mieux saisir l'évolution du théâtre contemporain, il est essentiel de tenir compte des changements qui se sont produits dans les autres arts.

\section{Cézanne, ou un nouveau moment de la perception}

$\mathrm{Si}$ "un nouveau style plastique implique l'apparition d'une nouvelle attitude de l'homme à l'égard du monde " ${ }^{20}$, l'œuvre de Cézanne a ouvert un nouveau chapitre de la peinture, ainsi qu'un nouveau moment de la perception. Située entre l'impressionnisme, auquel il participe d'abord, et le cubisme qu'il annonce dans sa maturité, sa position en fait un des peintres charnières qui vont contribuer à instaurer un nouveau langage plastique. Attaché d'abord aux recherches impressionnistes, il prend part au rejet de toute la tradition académique instaurée à la Renaissance, qui trouvait son fondement dans la croyance en la fixité de lois explicatives du monde extérieur. Dans ce mode traditionnel de création, "il était admis qu'un tableau constituait la représentation d'un fragment de la réalité (fixe ou mobile), saisie par un peintre

19 L'abduction est un concept utilisé par C. S. Peirce et Gregory Bateson pour signifier un certain type de raisonnement analogique. Chez Peirce, le terme recouvre essentiellement le mode inférentiel propre à la création, celui qui fonctionne par la formulation de nouvelles hypothèses sur le monde, se démarquant par là des modes inductifs et déductifs de raisonnement. Bateson, pour sa part, parle de l'abduction comme d'un mode de pensée similaire à la logique du rêve; ce mode fonctionne par le mixage de deux ou plusieurs éléments appartenant à un ordre de réalité différent, mais possédant des caractéristiques schématiques ou des composants abstraits similaires et donc susceptibles d'être associés dans l'esprit du créateur. L'association analogique de ces éléments hétérogènes va former une nouvelle entité qui agira à titre d'hypothèse novatrice et expérimentale sur le monde. 
spectateur, lui-même immobile "21. Le Cézanne impressionniste s'oppose d'abord à la peinture narrative. Ses investigations le mèneront progressivement vers une nouvelle façon de percevoir et de construire l'espace; sa peinture s'éloignera ainsi des sujets pour mettre en valeur les manières différentielles de les représenter; c'est ainsi que la montagne Sainte-Victoire, peinte selon divers points de vue, dérogera définitivement à la règle de l'immuabilité du monde extérieur. Cette démarche, qui écarte sa peinture d'une représentation plausible et lisible de la réalité, tient à ce qu'il part "du visible pour créer l'équivalent passionné des sensations" 22 . Par la mise en valeur de la "perspective vécue" au détriment de la perspective géométrique, Cézanne ouvre une série de recherches sur les sensations intimes et sur les modalités de la perception. À la sensation s'ajoute une démarche de composition - par le cône et les sphères - qui l'amènera à établir de nouvelles relations entre les fragments de sa perception. Dans la même foulée, Gauguin, Van Gogh et les cubistes démontreront que la projection linéaire ne donne pas une image exacte de ce que le peintre voit, que "ce que l'on appelle "vision normale" est simplement une vision sélectionnée et que le monde est infiniment plus riche en apparences qu'on ne l'a cru" ${ }^{23}$. Les cubistes tenteront également de suggérer l'essence du mouvement sur la toile, entre autre en introduisant la possibilité d'évoquer le déplacement de l'œil de l'observateur par rapport à un objet. Cette suggestion ne se veut pas une évocation du mouvement perçu "comme une réalité concrète et extérieure mais comme une expérience intérieure, personnelle, communicable par voie de signes intermédiaires" 24 . En définitive, Cézanne symbolise ce passage, continu de l'impressionnisme au cubisme - les deux pouvant être considérés comme participant à une même évolution de la pensée - du donné vers le construit, et donc d'un monde objectivé à un monde interprété subjectivement.

21 Michel Hoog, Cézanne "puissant et solitaire "Évreux, Découvertes Gallimard, 1989, p. 70.

22 Michel Hoog, ibid., p. 125.

23 Pierre Francastel, op. cit., p. 47.

24 Pierre Francastel, L'image, la vision et l'imagination: De la peinture au cinéma, Paris, Denoël/Gonthier, 1983, p. 173. 


\section{La perception a aussi une histoire...}

N'est-ce pas précisément à ce passage des sujets intéressants vers les modalités "vécues" de la perception auquel nous convie le texte de Normand Canac-Marquis? Aussi, l'abduction entre la peinture et le théâtre, élaborée dans Le syndrome de Cézanne, ne doit être comprise que dans le sens d'une transformation de la pensée artistique. On ne conçoit plus l'œuvre d'art comme un miroir de la réalité, mais comme un système capable de suggérer des sensations: "la notion de suggestion s'est substituée à celle de reproduction, celle de création conforme à l'ordre universel à celle d'imitation descriptive" "25. Le syndrome de Cézanne représente ce glissement de la pensée par une écriture et une structure "à la manière" de Cézanne. Le mode de pensée du protagoniste s'accomplit de façon cézanienne, ce qui témoigne bien du regard philosophique qu'insuffle la peinture à l'écriture relativement à notre mode actuel de représentation du monde. Nous retrouvons dans la pièce ce point de vue pictural par l'éloignement du sujet (il n'est reconstitué qu'à la fin du drame), la mise en valeur de la perspective vécue du protagoniste (ses états intérieurs), la multiplication des points de vue (l'accident peut être vu de trois façons différentes) et la composition à partir de fragments de réalité. Dans la littérature dramatique contemporaine surgit clairement le besoin de projection spatiale des sensations; cette dernière doit se lire à un second degré du texte, celui de la perspective architectonique; elle tente de suggérer un mouvement, celui de la conscience qui perçoit et interprète diversement le monde extérieur. De cette manière, la pièce offre une vision de l'être non plus "pensé en tant que fondement et stabilité de structures externes [majs] en tant qu'événement [...]: l'être n'est pas, comme le dit également Heidegger, il a lieu "26. Le syndrome de Cézanne met effectivement en scène une sorte de dialectique, d'où surgit l'impression de mouvement entre la pensée et les faits du monde extérieur. Comme Cézanne qui tenta d'imiter la "vision naturelle" et de "donner l'impression d'un ordre naissant, d'un objet en train d'apparaître, en train de s'agglomérer sous nos yeux" "27, Normand Canac-Marquis essaie de faire vivre le mouvement interne de per-

25 Ibid., p. 230.

26 Gianni Vattimo, La société transparente, Paris, Desclée de Brower, 1990, p. $97-98$.

27 Merleau-Ponty cité dans Cézanne -puissant et solitaire ", op. cit., p. 70. 
94

ception du protagoniste. Il n'y a pas de perception dans l'immobilité mais dans la seule mobilité. Le mode de composition du texte illustre cette nouvelle relation entre le sujet et le monde qui, loin d'être étanche, s'entrelace par diffraction.

À sa façon, Le syndrome de Cézanne s'éloigne radicalement du domaine de la causalité, là où la dramaturgie traditionnelle cherchait à justifier un acte ou un événement par une cause explicative. En découpant des fragments disparates de la réalité pour composer avec eux un montage à l'image du réel non plus externe mais interne - tout comme l'ont déjà fait Cézanne et les cubistes -, c'est la notion même de réel qui s'en trouve élargie; par l'introduction de la dimension psycho-sensorielle, la représentation artistique échappe à la détermination réaliste conventionnelle qui confinait l'acte créateur à une exploration du monde externe. C'est dans ce sens qu'il faut interpréter le début de la deuxième scène où il est écrit que "Cézanne représente la réalité en supprimant volontairement les règles classiques de la profondeur et des dimensions. Il oppose au réalisme une réalité différente qui donne au sujet sa raison profonde d'être". Ici, l'insistance est mise sur l'effet de conscience et sur la fabrication intérieure d'une image du monde; les fragments de réel s'ordonnent selon l'unité des perceptions créées dans l'esprit. Les nouvelles règles de composition développées dans Le syndrome de Cézanne démontrent bien qu'il n'y a pas suppression mais transfert, glissement du contenu vers un nouvel espace de compréhension. Comme l'a déjà fait observer Gregory Bateson, "la logique serait-elle un artefact se révélant inapproprié pour l'exploration des mécanismes internes de perception du monde?" 28

Le recours à la peinture de Cézanne comme modèle structurant d'un nouveau langage dramatique a une double portée. D'abord, cette nouvelle écriture dramatique prend part à l'ensemble des changements amorcés depuis le début du siècle dans les arts visuels en général, et dans la peinture en particulier; ce à quoi il convient d'ajouter l'importance grandissante qu'ont pris les arts mécaniques de reproduction du réel comme la photographie d'abord et le cinéma ensuite. Ces nouvelles formes et techniques artistiques témoignent de "la découverte de nouvelles structures intellectuelles reflétant des aspects encore inconnus de l'activité 
universelle "29. Pierre Francastel précise à ce propos: "la photographie a confirmé ceux qui recherchaient de nouveaux objets c'est-à-dire de nouveaux groupements possibles de sensations au lieu de leur donner l'impression que la nature brute leur fournissait d'elle-même le découpage. [...] Un peu plus tard, la découverte de l'image mobile [le cinéma] est venue encore accentuer cette évolution en insistant sur les possibilités ouvertes par le "montage" - dont Gauguin a tiré de merveilleux partis" 30. Ensuite, l'appel à la peinture met en valeur la relation essentielle entre les modalités sensorielles de perception et le langage de l'image. La pensée imagée, l'esprit qui pense par les images, est un phénomène intellectuel lié au mécanisme de la perception. Nous touchons là un des points fondamentaux qui définissent l'orientation du langage artistique contemporain, le texte theâtral inclu. Car, autant le modèle explicatif, narratif et rationnel correspond à des structures mentales d'appréhension du monde, autant le mécanisme de l'esprit qui pense par l'image doit être considéré comme un langage, comme un nouveau système de signification relié à de nouvelles formes de l'activité créatrice de l'esprit. Il s'agit de l'avènement d'un langage tendu vers l'introspection et le pouvoir d'association de l'esprit.

Autrement dit, l'art comme la technique participent aux transformations globales d'un système de pensée propre à une culture donnée; lorsque ce système atteint un certain niveau d'achèvement et de fixation, un changement d'échelle se produit, permettant à l'esprit humain d'envisager de nouvelles possibilités perceptives et intellectuelles. Ces possibilités se traduisent par l'apparition simultanée de nouvelles techniques et de nouveaux langages artistiques qui tous sont inclus dans une vision similaire, c'est-à-dire dans l'enregistrement de nouveaux rapports entre objets et sujets.

Si le théâtre de l'image fait référence aux modalités de la perception, qui se communiquent moins de façon linéaire et logique que par un langage analogique, alors il existe aussi un texte de l'image. Mais, comme nous avons tenté de le montrer avec Le syndrome de Cézanne, il faudra apprendre à lire ces pièces au second degré, c'est-à-dire dans les biais architectoniques offerts

29 Pierre Francastel, Limage, la vision et limagination, op. cit., p. 188.

30 Pierre Francastel, Peinture et société, op. cit., p. 161-162. 
96

par celles-ci, le sens "imagé "et donc perceptif surgissant dans de plus vastes unités du message. Et le texte fait image parce qu'il s'ancre de plus en plus dans les formes générales de l'expérience sensible. Ainsi, le spectateur n'est plus mis face à l'objet artistique, mais au centre, entre le monde référentiel externe et le monde fabriqué par l'artiste; de ces deux représentations, il doit tenter de se composer une vision propre. Son travail d'interprétation devient ainsi similaire à celui de la conscience qui enregistre et qui traite les multiples images du monde. Le théâtre existeraitil, entre autre, pour permettre à notre esprit de s'adapter à un monde qui se complexifie sans cesse? 